\title{
SOLUTIONS OF THE DIFFUSION EQUATION IN CONES AND WEDGES
}

\author{
E. M. Rodriguez Ventura, ${ }^{1}$ M. M. R. Williams ${ }^{2}$ and J. Wood ${ }^{1}$ \\ ${ }^{1}$ Nuclear Engineering Department, Queen Mary College, University of London, Mile End Road, \\ London E1 4NS U.K. and ${ }^{2}$ Nuclear Engineering Department, The University of Michigan, \\ Cooley Building, N. Campus, Ann Arbor, MI 48109-2104, U.S.A.
}

(Received 1 August 1987; in revised form 29 September 1987)

\begin{abstract}
The neutron diffusion equation has been solved for cones and finite wedges. Criticality conditions are derived linking the dimensions of the cones and wedges to the nuclear properties of the system. The fundamental mode flux is evaluated numerically for a number of cases. We have also discovered an interesting minimum in the leakage rate as the cone or wedge angle passes through a certain value for a fixed volume. Some practical implications of this result are pointed out.

The exact solutions derived have been used as benchmarks to assess the accuracy of the finite element code TRIPAC in $R-Z$ and $X-Y-Z$ geometries for a four-group criticality problem in cones and wedges. Excellent agreement is found between the analytical results and the code for both eigenvalues and fluxes.
\end{abstract}

\section{INTRODUCTION}

Solutions of the diffusion equation in nonstandard geometries are of intrinsic interest but also of practical value since the results may be used to check the accuracy of computer codes. With this in mind we present some solutions of the equation (Glastone and Edlund, 1953):

$$
\nabla^{2} \phi+B^{2} \phi=0,
$$

subject to zero flux on the boundary for nonreentrant cones and wedges.

Our solutions are given analytically and we present the flux distribution and the critical condition in terms of the physical dimensions of the cone or wedge. These results are employed to obtain a comparison with results from the finite element code TRIPAC and lend further support to its accuracy.

In addition to obtaining analytical solutions, we are able to show that, for a given buckling, the critical cone volume and corresponding surface area exhibit a minimum for a certain value of the cone angle. Similarly, for a fixed critical volume, the buckling has a minimum at a particular cone angle. Analogous behaviour arises for wedges.

\section{SOLUTION OF THE DIFFUSION EQUATION FOR A BARE CONE}

\subsection{General solution and eigenvalues}

Following the usual procedure (Morse and Feshbach, 1953), we can write the Helmholtz equation (called the diffusion equation in reactor physics) in spherical coordinates as:

$$
\frac{1}{r^{2}} \frac{\partial}{\partial r}\left(r^{2} \frac{\partial \phi}{\partial r}\right)+\frac{1}{r^{2} \sin \theta} \frac{\partial}{\partial \theta}\left(\sin \theta \frac{\partial \phi}{\partial \theta}\right)+B^{2} \phi=0,
$$

where we note that $\phi=\phi(r, \theta), \theta$ being the angle measured with respect to the $z$-axis. There is no dependence on the azimuthal angle due to symmetry.

The boundary conditions are:

(a) the flux is everywhere finite,

(b) $\phi\left(r, \theta_{0}\right)=0$,

(c) $\phi\left(R_{0}, \theta\right)=0$,

where $R_{0}$ is the cone radius and $\theta_{0}$ is its half-angle. We restrict $\theta_{0}<\pi / 2$ because we wish to avoid reentrant conditions. However, values of $\theta_{0}>\pi / 2$ could have physical significance if it is assumed that the volume contained within the reentrant line-of-sight region is black to neutrons.

Using the standard procedure of separation of variables, we can write the solution to equation (2) which satisfies condition (3) as :

$$
\phi(r, \theta)=\frac{\mathrm{A}}{\sqrt{r}} J_{v+1 / 2}(B r) P_{v}(\cos \theta),
$$

where $\mathrm{A}$ is an arbitrary constant and $v$ is a parameter to be determined. $J_{v+1 / 2}(Z)$ is a Bessel function and $P_{v}(\cos \theta)$ is a Legendre polynomial. 
Boundary condition (4) leads to:

$$
P_{v}\left(\cos \theta_{0}\right)=0 ; \text { all } v \geqslant 0 .
$$

The roots of this equation lead to the appropriate value of $v$. In fact unless $v$ is an integer there is an infinite number of solutions, but we are interested only in the smallest root since this corresponds to the fundamental mode. The precise roots of equation (7) require some effort to obtain but a good first approximation arises from the following asymptotic representation, viz. (Abramowitz and Stegun, 1965):

$$
\begin{aligned}
P_{v}(\cos \theta) \sim\left[\frac{2}{\pi \sin \theta}\right]^{1 / 2} \frac{\Gamma(v+1)}{\Gamma\left(v+\frac{3}{2}\right)} & \\
& \quad \times \sin \left\{\left(v+\frac{1}{2}\right) \theta+\frac{\pi}{4}\right\}+O\left(v^{-3 / 2}\right),
\end{aligned}
$$

where $v \geqslant 1$ and $\theta$ is confined to the interval $(\varepsilon, \pi-\varepsilon)$ where $\varepsilon$ is a small number. The greater the value of $\theta$, the more accurate is equation (8).

Neglecting the term of $O\left(v^{-3 / 2}\right)$ and applying condition (7) we find

$$
\left(v+\frac{1}{2}\right) \theta_{0}+\frac{\pi}{4}=n \pi, \quad n=0,1,2, \ldots
$$

The fundamental mode arises when $n=1$ ( $n=0$ corresponds to negative $v$ ) and thus:

$$
v=\frac{3 \pi}{4 \theta_{0}}-\frac{1}{2} \text {. }
$$

By comparison with an exact calculation, it may be shown that this formula is accurate to better than $2 \%$ even for $\theta_{0}=1.8^{\circ}$. It becomes progressively more accurate as $\theta_{0}$ increases being exact for $\theta_{0}=\pi / 2$.

The flux can now be written as :

$$
\phi(r, \theta)=\frac{\mathrm{A}}{\sqrt{r}} J_{\frac{3 \pi}{4 \theta_{0}}}(B r) P_{\frac{3 \pi}{4 \theta_{0}}-\frac{1}{2}}(\cos \theta) .
$$

Finally, using equation (5), we have :

$$
J_{\frac{3 \pi}{4 \theta_{0}}}\left(B R_{0}\right)=0,
$$

which yields the critical condition between $B$ and the physical dimensions of the cone.

The roots of equation (12) have been found from tables of Bessel functions (Abramowitz and Stegun, 1965) and are listed in Table 1a. There is an infinite number of such roots for a given $\theta_{0}$ but we are only concerned with the one that corresponds to a nonnegative eigenfunction. A useful empirical relation between $B R_{0}$ and $\theta_{0}$ is found to be :

$$
B R_{0}=3.0725 \theta_{0}^{-0.9415}+2.5076 .
$$

Table 1a. Zeros of Bessel function of first kind of order $3 \pi / 4 \theta_{0}$

\begin{tabular}{cccc}
\hline $3 \pi / 4 \theta_{0}$ & $\theta_{0}$ & $\left(B R_{0}\right)$ exact & $\left(B R_{0}\right)$ approx. \\
\hline $3 / 2$ & $\pi / 2$ & 4.493409 & 4.516 \\
2 & $3 \pi / 8$ & 5.135620 & 5.141 \\
$5 / 2$ & $3 \pi / 10$ & 5.763459 & 5.756 \\
3 & $\pi / 4$ & 6.380160 & 6.365 \\
$7 / 2$ & $3 \pi / 14$ & 6.987932 & 6.967 \\
$9 / 2$ & $\pi / 6$ & 8.182561 & 8.158 \\
$11 / 2$ & $3 \pi / 22$ & 9.355812 & 9.333 \\
6 & $\pi / 8$ & 9.936110 & 9.915 \\
7 & $3 \pi / 28$ & 11.08637 & 11.072 \\
8 & $3 \pi / 32$ & 12.22509 & 12.220 \\
$25 / 2$ & $3 \pi / 50$ & 17.250455 & 17.291 \\
$39 / 2$ & $3 \pi / 78$ & 24.878005 & 24.979 \\
\hline
\end{tabular}

The accuracy of this relationship is better than $0.5 \%$ throughout the range of values of $\theta_{0}$ shown in Table 1.

As an indicator of the overall accuracy of equations (10) and (13) we note that an accurate determination of $B R_{0}$ for $\theta_{0}=1.8^{\circ}$ leads to $B R_{0}=80.53$, whilst the approximation yields $B R_{0}=82.39$, an error of $2.3 \%$. As $\theta_{0}$ increases, this error progressively becomes smaller, e.g. at $\theta_{0}=13.22^{\circ}$, the error is $2.1 \%$ and at $\theta_{0}=54.73^{\circ}$ it is $-0.7 \%$. Thus our empirical result would appear to be a useful approximation for bare cones.

In some calculations, it is more practical to select an integer value of $v$ and find the corresponding value of $\theta_{0}$ from the equation:

$$
P_{v}\left(\cos \theta_{0}\right)=0 .
$$

Such roots are simply the Gauss-Legendre quadrature points and are known very accurately. With an integer value of $v$ it is easy to find the roots of

$$
J_{v+1 / 2}\left(B R_{0}\right) \text {, }
$$

from the tables in Abramowitz and Stegun (1965). We shall adopt this procedure in our comparison with the finite element method to be described below and give some illustrative results in Table $1 \mathrm{~b}$.

Table 1b. Exact roots and half-angles for cone

\begin{tabular}{ccl}
\hline$v$ & \multicolumn{1}{c}{$B R_{0}$} & \multicolumn{1}{c}{$\theta_{0}$} \\
\hline 1 & 4.493409 & 90 \\
2 & 5.763459 & 54.73561 \\
3 & 6.987932 & 39.23152 \\
4 & 7.58834 & 30.55559 \\
5 & 8.77148 & 25.01733 \\
6 & 9.93611 & 21.17690 \\
7 & 11.08637 & 18.35785 \\
8 & 12.22509 & 16.20078 \\
\hline
\end{tabular}




\subsection{Optimum size of cone}

Let us assume that we have a cone of fixed buckling, i.e. fixed net leakage, and require to know how the volume and surface area vary with $\theta_{0}$.

The volume of a cone is given by the expression :

$$
V=\frac{2 \pi R_{0}^{3}}{3}\left(1-\cos \theta_{0}\right) \text {. }
$$

Using the empirical relationship (13) for $R_{0}$ as a function of $\theta_{0}$ (remember that $B$ is fixed), we find that the volume is :

$$
V\left(\theta_{0}\right)=\frac{2 \pi}{3 B^{2}}\left(\alpha \theta_{0}^{-\beta}+\gamma\right)^{3}\left(1--\cos \theta_{0}\right)
$$

where $\alpha, \beta$ and $\gamma$ are given in (13).

It is readily shown that $V\left(\theta_{0}\right)$ has a minimum at $\theta_{0}=0.5259\left(30.132^{\circ}\right)$. Similarly, the surface area of the cone which is given by:

$$
S\left(\theta_{0}\right)=2 \pi R_{0}^{2}\left(1-\cos \theta_{0}\right)+\pi R_{0}^{2} \sin \theta_{0}
$$

also has a minimum at $\theta_{0}=0.559\left(32^{\circ}\right)$. Both $V\left(\theta_{0}\right)$ and $S\left(\theta_{0}\right)$ are shown in Fig. 1 as functions of $\theta_{0}$.

Let us now consider a cone of constant volume and examine the buckling as a function of $\theta_{0}$. Using the earlier formulae we readily find that :

$$
B^{2}\left(\theta_{0}\right)=\left(\frac{2 \pi}{3 V}\right)^{2 / 3}\left(\alpha \theta_{0}^{-\beta}+\gamma\right)^{2}\left(1-\cos \theta_{0}\right)^{2 / 3}
$$

This is illustrated graphically in Fig. 2 and exhibits a minimum at $\theta_{0} \simeq \pi / 6$. Clearly there is a critical value of cone angle which minimizes the leakage. The possibility of using such a phenomenon as a reactor control device should be noted.

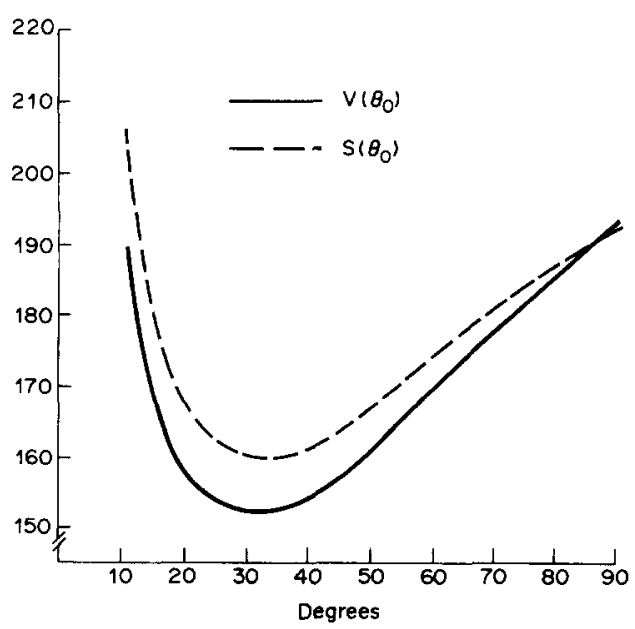

Fig. 1. The variation of the critical volume and surface area with cone half-angle $\theta_{0}$; the ordinate is in arbitrary units.

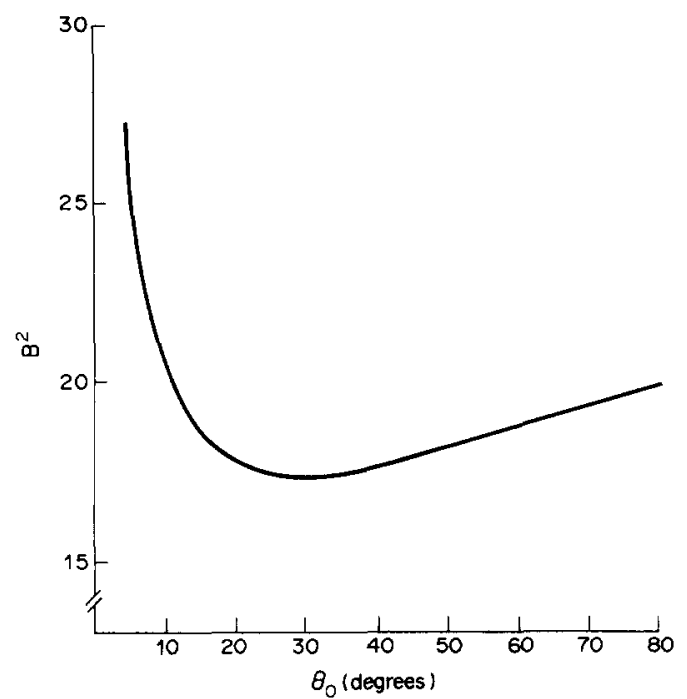

Fig. 2. The variation of the buckling with cone half-angle, $\theta_{0}$, for a fixed volume; the ordinate is in arbitrary units.

\subsection{Flux distribution in a cone}

To illustrate the flux shape in the cone, we consider the following parameters: $\theta_{0}=\pi / 6\left(30^{\circ}\right)$ and hence $B R_{0}=8.182561$, from Table 1 . We also note that $v=4$ and thus the flux distribution is from equation (6) :

$$
\phi(r, \theta)=\left(\frac{\pi}{2 B r}\right)^{1 / 2} J_{9 / 2}(B r) P_{4}(\cos \theta)
$$

The flux is given in Table 2 for $\theta=0$ for various values of the ratio $r / R_{0}$. We note that a maximum arises at $r=0.685 R_{0}$. It is also interesting to examine the angular variation of the flux which follows the function $P_{4}(\cos \theta)$. The values of $P_{4}(\cos \theta)$ for a range of

Table 2. Flux along central axis of cone,

\begin{tabular}{ll}
\multicolumn{2}{c}{$\theta_{0}=\pi / 6, B R_{0}=8.18$} \\
\hline$v / R_{i)}$ & $\phi(r, 0)$ \\
\hline 1 & 0 \\
0.917 & 0.0793 \\
0.856 & 0.1327 \\
0.733 & 0.1968 \\
0.685 & 0.2015 \\
0.672 & 0.2008 \\
0.611 & 0.1870 \\
0.489 & 0.1249 \\
0.367 & 0.05615 \\
0.244 & 0.01408 \\
0.122 & $1.011 \times 10$ \\
0.0611 & $6.539 \times 10$ \\
0.0 & 0.0 \\
\hline
\end{tabular}


Table 3. Angular flux across cone

\begin{tabular}{ll}
\hline \multicolumn{1}{c}{$\theta$} & \multicolumn{1}{c}{$P_{4}(\cos \theta)$} \\
\hline 0.0 & 1.0 \\
5 & 0.9623 \\
10 & 0.8532 \\
20 & 0.4750 \\
25 & 0.2465 \\
30 & 0.0234 \\
30.55559 & 0.0
\end{tabular}

$\theta$ are shown in Table 3 . The flux is not precisely zero at $\theta=30^{\circ}$ due to the approximate formula for $v$ of equation (10). In fact, it is zero at the smallest root of $P_{4}(\cos \theta)=0$, i.e. $\theta=30.55559^{\circ}$. The fluxes are illustrated graphically in Fig. 3.

\section{SOLUTION OF THE DIFFUSION EQUATION FOR A BARE, FINITE WEDGE}

\subsection{General solution and eigenvalues} is:

The diffusion equation for a wedge of finite height

$$
\frac{1}{r} \frac{\partial}{\partial r}\left(r \frac{\partial \phi}{\partial r}\right)+\frac{1}{r^{2}} \frac{\partial^{2} \phi}{\partial \theta^{2}}+\frac{\partial^{2} \phi}{\partial z^{2}}+B^{2} \phi=0
$$

where $\phi=\phi(r, \theta, z)$.

We assume that one face of the wedge is in the plane $\theta=0$ and the other in the plane $\theta=\theta_{0}$. The wedge is

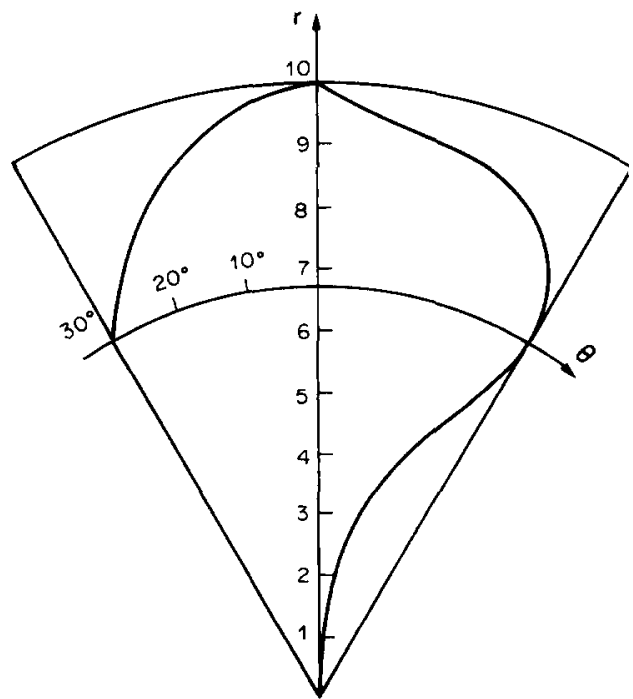

Fig. 3. Flux distribution inside a cone with $\theta_{0}=\pi / 6, R_{0}=10$. The curve on the right hand side of the figure represents the radial flux distribution and that on the left the angular distribution ; actual magnitudes are arbitrary. of radius $R_{0}$ and height $H$. The zero flux boundary conditions are:

$$
\begin{gathered}
\phi(r, 0, z)=0 \\
\phi\left(r, \theta_{0}, z\right)=0 \\
\phi\left(R_{0}, \theta, z\right)=0 \\
\phi(0, \theta, z)=0 \\
\phi(r, \theta, 0)=0 \\
\phi(r, \theta, H)=0 .
\end{gathered}
$$

Using separation of variables, we find that the solution can be written :

$$
\phi(r, \theta, z)=C J_{\mu_{1}}\left(B_{r} r\right) \sin \left(\mu_{1} \theta\right) \sin \left(B_{z} z\right),
$$

where

$$
\mu_{1}=\pi / \theta_{0}
$$

and

$$
B_{z}^{2}=\left(\frac{\pi}{H}\right)^{2}=B^{2}-B_{r}^{2} .
$$

$B_{r}^{2}$ is the radial buckling and is found from condition (23) viz.

$$
J_{\pi / \theta_{0}}\left(B_{r} R_{0}\right)=0 .
$$

Thus we have a relation between the physical dimensions of the wedge and its nuclear properties, $B^{2}$. From tables of zeros of Bessel functions we can obtain Table 4. Again, for convenience in calculation, an empirical relationship between $B_{r} R_{0}$ and $\theta_{0}$ has been obtained thus :

$$
B_{r} R_{0}=4.0488 \theta_{0}^{-0.9378}+2.50 .
$$

Table 4. Zeros of Besssel function of first kind of order, $\pi / \theta_{0}$

\begin{tabular}{cccc}
\hline$\pi / \theta_{0}$ & $\theta_{0}$ & $\left(B_{r} R_{0}\right)$ exact & $\left(B_{r} R_{0}\right)$ approx. \\
\hline 1 & $\pi$ & 3.83171 & 3.884 \\
3.2 & $2 \pi / 3$ & 4.493409 & 4.524 \\
2 & $\pi / 2$ & 5.135620 & 5.151 \\
$5 / 2$ & $2 \pi / 5$ & 5.763459 & 5.768 \\
3 & $\pi / 3$ & 6.380160 & 6.377 \\
$7 / 2$ & $2 \pi / 7$ & 6.987932 & 6.981 \\
4 & $\pi / 14$ & 7.58834 & 7.578 \\
$9 / 2$ & $2 \pi / 9$ & 8.182561 & 8.171 \\
5 & $\pi / 5$ & 8.77148 & 8.760 \\
$11 / 2$ & $2 \pi / 11$ & 9.355812 & 9.346 \\
6 & $\pi / 6$ & 9.936110 & 9.928 \\
$13 / 2$ & $2 \pi / 13$ & 10.512835 & 10.507 \\
7 & $\pi / 7$ & 11.08637 & 11.083 \\
$15 / 2$ & $2 \pi / 15$ & 11.657032 & 11.657 \\
8 & $\pi / 8$ & 12.22509 & 12.228 \\
$25 / 2$ & $2 \pi / 25$ & 17.250455 & 17.284 \\
$39 / 2$ & $2 \pi / 39$ & 24.878005 & 24.933 \\
\hline
\end{tabular}


The accuracy of the expression may be judged from Table 4. For $\theta_{0}<120^{\circ}$ it is better than $0.7 \%$.

\subsection{Optimum size of wedge}

As in the case of the cone, we look for an angle which minimizes the buckling for a given wedge volume. Using equation (30) and the volume of the wedge, we get :

$$
B_{r}^{2}\left(\theta_{0}\right)=\frac{H}{2 V} \theta_{0}\left(a \theta_{0}^{-b}+c\right)^{2},
$$

where $a, b$ and $c$ are defined by equation (30). As Fig. 4 shows, the radial buckling has a minimum and by differentiation of equation (31) we find:

$$
\left(\theta_{0}\right)_{\min }=\left\{\frac{a(2 b-1)}{c}\right\}^{1 / b}=83.15^{\circ} .
$$

\subsection{Flux distribution in a wedge}

For $\theta_{0}=\pi / 4$, we find that the flux distribution can be written:

$$
\phi(r, \theta, z)=J_{4}\left(B_{r} r\right) \sin (4 \theta) \sin \left(\frac{\pi z}{H}\right)
$$

where $B_{z}=\pi / H$ and $B_{r} R_{0}=7.58834$.

The maximum value of $\phi$ along the radial direction arises when:

$$
\frac{\mathrm{d} J_{4}(B, r)}{\mathrm{d} r}=0
$$

which leads to $B_{r} r_{\max }=5.31755$, i.e. $r_{\max }=0.70075 R_{0}$. Of course, in the axial direction, the maximum is at $z=H / 2$ and in the $\theta$-direction at $\theta_{0} / 2$.

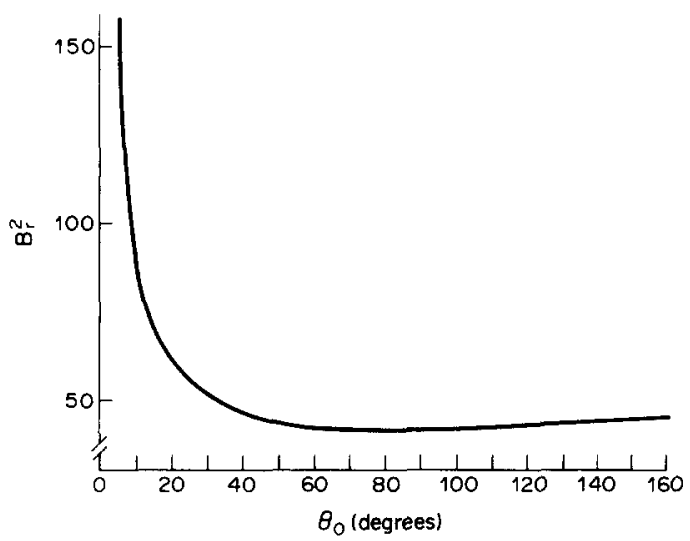

Fig. 4. Variation of radial buckling $B_{r}^{2}$ with wedge angle $\theta_{0}$ for constant volume; the ordinate is in arbitrary units.

\section{NUMERICAL VERIFICATION OF CODE AND THEORY}

To verify the theoretical relationships derived in the previous sections, particular examples of the cone and wedge configurations are solved by means of a multigroup finite element transport code called TRIPAC. This code is a development of two earlier codes, namely FELICIT (Wood and Williams, 1984; Wood, 1986) and EVENT (De Oliveira, 1986, 1987). A feature of TRIPAC, as of its precursors, is its ability to handle complex geometries, and the two configurations considered in this paper provide an interesting test of this aspect of the code's capabilities. The numerical solutions that we give also provide useful benchmarks for proving other techniques and computer codes for which the claim of geometrical flexibility is also advanced. In this paper we are concerned with diffusion theory, i.e. $P_{1} / P_{1}$ solutions, but of course TRIPAC is also capable of providing higherorder transport solutions, namely, $P_{n} / P_{l}$.

Two cases of the cone are considered, and two of the wedge. Four-group linear anisotropic scattering data is used for the homogeneous systems. The data is based on the simple HTGR core data used by White and Frank (1987). Our data is listed in Appendix A; the corresponding value of materials buckling is $B_{\max }^{2}=1.9701604 \times 10^{-2}$. In the computations, iterations are continued until the eigenvalue $\left(k_{\text {eff }}\right)$ and eigenvector have converged, respectively, to $10^{-6}$ and $10^{-5}$. Also the eigenvector is normalized to unit fission neutrons produced in the system, i.e.

$$
\frac{1}{k_{\mathrm{efr}}} \iint \mathrm{d} E \mathrm{~d} v \bar{v} \Sigma_{f} \phi(E, \mathbf{r})=1 .
$$

In the case of the wedge, the eigenvalue is actually normalized to $1 / 4$ of the total volume-this is explained below. It is perhaps worth mentioning that this particular data has the fundamental and next lower eigenvalue close together (White and Frank, 1987). Thus, unless a power iteration convergence acceleration scheme is employed in the code, a large number of iterations may be necessary to produce the accuracy we quote in TRIPAC, outer iteration acceleration is achieved by Chebyshev extrapolation.

\subsection{The cone}

The cone is solved in $R-Z$ geometry using 81 quadratic elements. The element mesh employed is illustrated in Fig. 5. The exact and TRIPAC results are compared in Tables 5 and 6 . Clearly, the agreement is excellent. The flux distribution along the $z$-axis of the smaller cone is shown in Fig. 6.

Figure 7 shows the variation of critical volume of a cone for fixed buckling as a function of the cone 

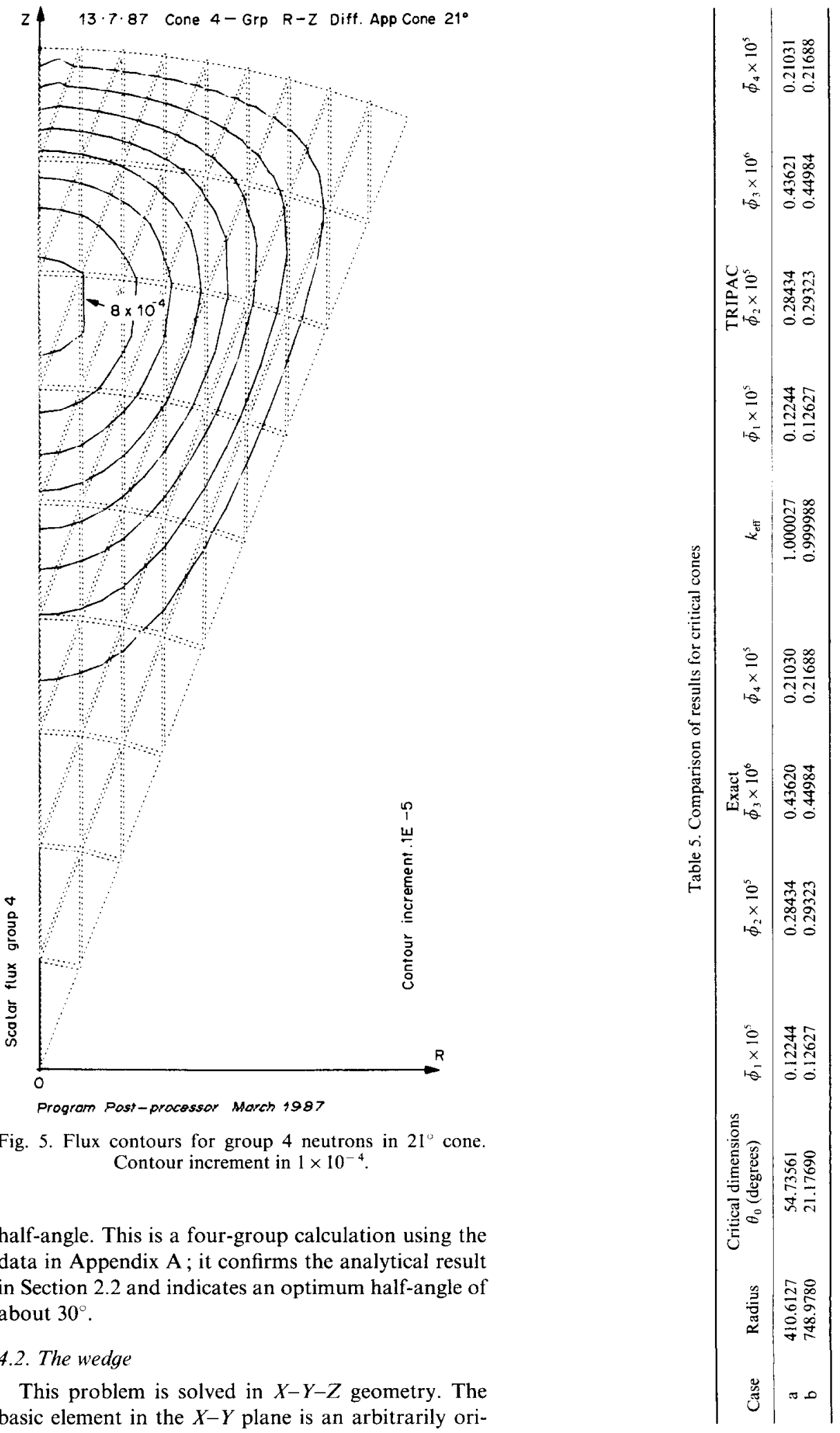

Fig. 5. Flux contours for group 4 neutrons in $21^{\circ}$ cone. Contour increment in $1 \times 10^{-4}$.

half-angle. This is a four-group calculation using the data in Appendix A ; it confirms the analytical result in Section 2.2 and indicates an optimum half-angle of about $30^{\circ}$.

\subsection{The wedge}

This problem is solved in $X-Y-Z$ geometry. The basic element in the $X-Y$ plane is an arbitrarily ori- 
Table 6. Comparison of critical flux distributions in $54^{\circ}$ cone, group 4 neutrons

\begin{tabular}{crcc}
\hline$r$ & $z$ & TRIPAC $\times 10^{5}$ & EXACT $\times 10^{5}$ \\
\hline 0.0 & 390.082 & 0.1324 & 0.1294 \\
0.0 & 307.960 & 0.6317 & 0.6271 \\
0.0 & 287.429 & 0.7155 & 0.7116 \\
0.0 & 246.368 & 0.7991 & 0.7970 \\
0.0 & 184.776 & 0.7031 & 0.7020 \\
0.0 & 123.184 & 0.4173 & 0.4173 \\
0.0 & 61.592 & 0.1234 & 0.1231 \\
95.777 & 248.827 & 0.6213 & 0.6208 \\
126.706 & 160.352 & 0.3215 & 0.3215 \\
152.234 & 336.739 & 0.1960 & 0.1969 \\
\hline
\end{tabular}

Note: cone is solved by TRIPAC in $R-Z$ geometry.

ented triangle. Again, a quadratic triangle is used. The subdivision of the $X-Y$ plane is shown in Fig. 8. These triangles form the cross sections of prisms whose axes are parallel to the $z$-axis. Each prism so formed is further subdivided into 3 tetrahedra. Thus the basic element in space is, in this case, a quadratic tetrahedron (with 10 nodes). Because of symmetry, the problem need only be solved in a $1 / 4$ wedge by taking the origin to be at the mid-height of the wedge and exploiting symmetry boundary conditions along appropriate axes. As with the cone, the boundary condition on the surface of the wedge is zero flux.

The exact and TRIPAC results are compared in Tables 7 and 8 . In TRIPAC, 735 quadratic tetrahedra are used for each wedge. An isometric view of the flux in a critical wedge is shown in Fig. 9. For the wedge, as for the cone, the agreement between theory and TRIPAC results is excellent.

Appendix $B$ indicates how the volume-averaged fluxes are calculated for cones and wedges.

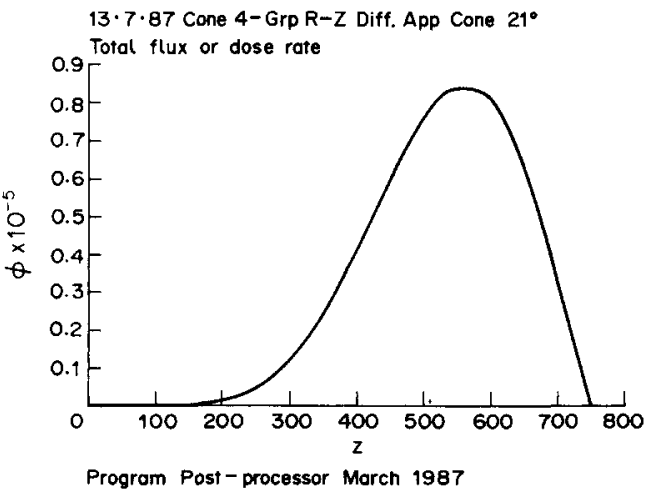

Fig. 6. Variation of flux along $z$-axis for $21^{\circ}$ cone (group 4 neutrons). 


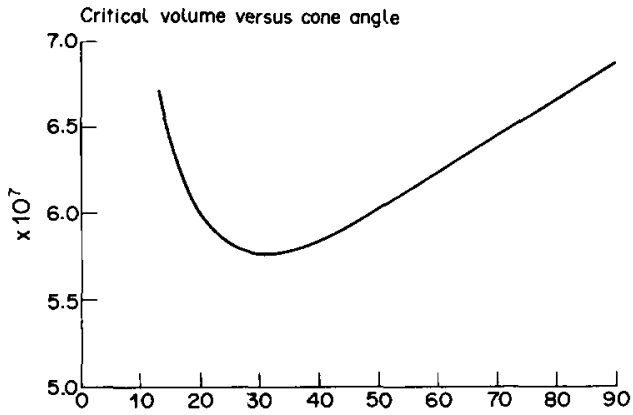

Fig. 7. Variation of critical volume of cone with half-angle $\theta_{0}$ for fixed buckling using four-group data.

\section{CONCLUSIONS AND SUMMARY}

Some exact solutions of the diffusion equation in cones and wedges have been obtained leading to critical conditions and fundamental mode flux distributions. We have observed that the volume and surface area of cones and wedges go through a minimum at a certain value of cone or wedge angle for a fixed buckling. Similarly, the buckling has a minimum for

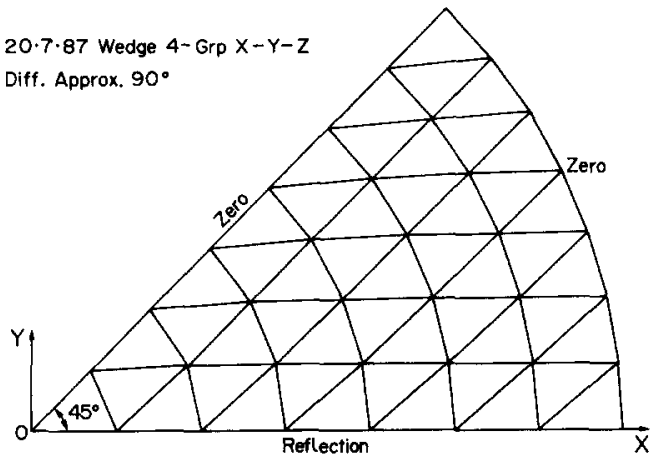

GEM VI 1.9.86

Fig. 8. Subdivision of $X-Y$ plane into quadratic triangles for $90^{\circ}$ wedge. Because of symmetry, only $1 / 2$ of $X-Y$ plane need be solved and $1 / 4$ of volume of wedge.

fixed volume at a critical angle. The latter condition implies a minimum leakage condition and has implications for reactor control and storage of fissile material.

We have also used these exact solutions to assess the accuracy of the TRIPAC finite element code. A

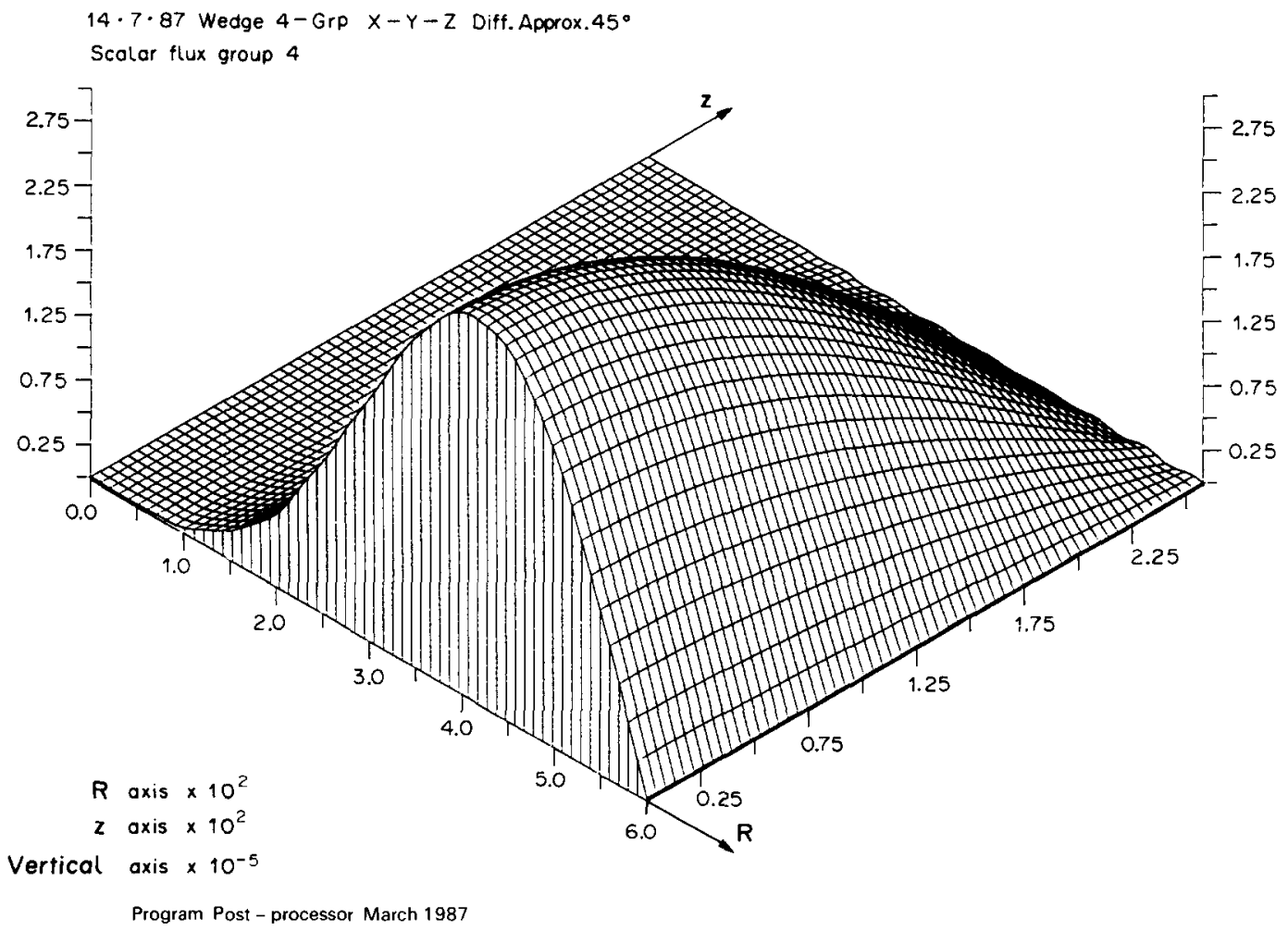

Fig. 9. Isometric view of flux in $45^{\prime}$ wedge, for the plane $Y=0$ (group 4 neutrons). 
Table 8. Comparison of critical flux distributions in $90^{\circ}$ wedge, group 4 neutrons

\begin{tabular}{ccccc}
\hline \multicolumn{1}{c}{$x$} & $y$ & $z$ & TRIPAC $\times 10^{5}$ & EXACT $\times 10^{5}$ \\
\hline 85.714 & 0.0 & 0.0 & 0.3324 & 0.3370 \\
171.429 & 0.0 & 0.0 & 1.1782 & 1.1737 \\
257.143 & 0.0 & 0.0 & 2.0833 & 2.0712 \\
342.857 & 0.0 & 0.0 & 2.5521 & 2.5390 \\
428.571 & 0.0 & 0.0 & 2.2798 & 2.2733 \\
514.286 & 0.0 & 0.0 & 1.3032 & 1.3072 \\
190.536 & 97.0872 & 70.6011 & 0.6853 & 0.6826 \\
414.445 & 99.740 & 84.7213 & 1.1970 & 1.1958 \\
355.528 & 308.062 & 0.0 & 0.2642 & 0.2666 \\
300.917 & 160.844 & 70.6011 & 0.9993 & 0.9964 \\
\hline
\end{tabular}

Note : wedge is solved by TRIPAC in $X-Y-Z$ geometry.

four-group bare reactor criticality problem is solved for a variety of wedges and cones. Both eigenvalues and critical fluxes are shown to be in excellent agreement with the analytical results. Cones and wedges are a sensitive test of any numerical procedure and lend further confidence in the accuracy of the TRIPAC code.

\section{REFERENCES}

Abramowitz M. and Stegun I. A. (1965) Handbook of Mathematical Functions. Dover, New York.

Glasstone S. and Edlund M. (1953) Nuclear Reactor Theory, Van Nostrand, New York.

Morse P. M. and Feshbach H. (1953) Methods of Theoretical Physics. McGraw-Hill, New York.

White J. R. and Frank B. R. (1987) International Topical Meeting on Advances in Reactor Physics, Mathematics and Computation, Vol. 3, p. 1385. American Nuclear Society.

Wood J. and Williams M. M. R. (1984) Prog. Nucl. Energy $14,21$.

Wood J. (1986) Ann. nucl. Energy 13, 91.

De Oliveira C. R. E. (1986) Ann. nucl. Energy 13, 227.

De Oliveira C. R. E. (1987) Ph.D. Thesis, University of London.

\section{APPENDIX A}

For each energy group $i$ :

$$
\begin{array}{ccc}
\Sigma & \Sigma_{\alpha} & \bar{v} \Sigma_{f} \\
\Sigma_{s 0}\left(E_{i} \rightarrow E_{1}\right) & \Sigma_{s 0}\left(E_{i} \rightarrow E_{2}\right) \\
\Sigma_{s 1}\left(E_{i} \rightarrow E_{1}\right) & \Sigma_{s 1}\left(E_{i} \rightarrow E_{2}\right)
\end{array}
$$

Note : diffusion coefficient $D=1 / 3 \Sigma_{t r}$ where $\Sigma_{t r}=\Sigma-\Sigma_{s 1}$.

Four-group data

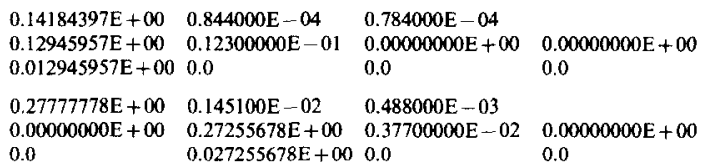

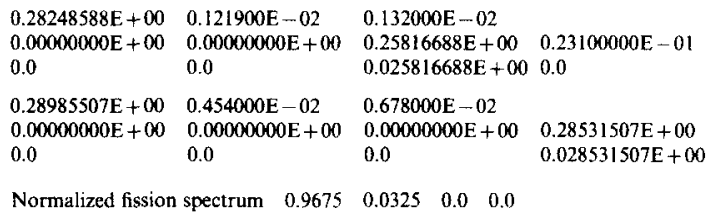

\section{APPENDIX B}

\section{Average Fluxes}

In order to obtain average fluxes it is necessary to calculate :

$$
\bar{\phi}=\frac{1}{V} \int_{V} \mathrm{~d} \mathbf{r} \phi(\mathbf{r}) .
$$

For the cone with:

$$
\phi(r, \theta)=\frac{1}{\sqrt{r}} J_{v+1 / 2}\left(B_{r}\right) P_{r}(\cos \theta),
$$

this expression becomes:

$$
\begin{aligned}
\phi=\frac{3}{R_{c}^{3}\left(1-\cos \theta_{0}\right)} \int_{0}^{R_{c}} \mathrm{~d} r r^{3 / 2} J_{v+1 / 2}\left(B_{r}\right) & \\
& \left.\times \int_{0}^{\theta_{0}} \mathrm{~d} \theta \sin \theta P_{v} \cos \theta\right) .
\end{aligned}
$$

For integer values of $v$, it is easy to carry out the $\theta$ integration and although the integration over the Bessel function can be represented by an infinite sum, it is more convenient to evaluate this directly by quadratures.

For wedges we have

$$
\phi(r, \theta, z)=J_{\mu_{1}}\left(B_{r} r\right) \sin \left(\mu_{1} \theta\right) \sin \left(B_{z} z\right),
$$

whence

$$
\Phi=\frac{8}{\pi^{2}\left(B_{r} R_{0}\right)^{2}} \int_{0}^{B_{r} R_{0}} \mathrm{~d} x x J_{\mu_{1}}(x),
$$

where

$$
\mu_{1}=\pi / \theta_{0} .
$$

